\title{
TITLE: THORACIC EPIDURAL OR BETA-BLOCKERS DO NOT REDUCE POST OPERATIVE MI
}

AUTHORS: W. Scott Beattie, PhD, MD, Duminda N Wijeysundera MD, Keyvan Karkouti MSc. MD, Stuart McCluskey PhD MD.

AFFILIATION: Department of Anesthesia \& Pain Management, Toronto General Hospital, UHN, University of Toronto, 200 Elizabeth Street, Toronto, ON, Canada M5G 2C4

INTRODUCTION: Myocardial infarction (POMI) is the leading cause of morbidity after non-cardiac surgery. Beta -blockers (BB) are recommended to reduce POMI but recent evidence suggests that the effect may be minimal. Meta-analysis of thoracic epidural analgesia (EPI) suggest a major reduction in POMI but this has not been tested in a large enough randomized trial. There is no data on the relative effects of these two modalities.

METHODS: Following REB approval our prospective pain database detailed pre-operative risk factors, post-operative medications, analgesic management and pain outcomes. All epidurals in this analysis were thoracic. We searched our database for discharge ICD 9 codes and all post-op troponin I levels. We defined a MI as troponin > 0.6. Post-op beta blockade was defined as the use of any beta blocker with in the first 48 hours. A preoperative Revised Cardiac Risk Index (RCRI) was calculated for each patient. We performed multivariate analysis for the pre-operative predictors of POMI and BB and EPI. In addition the relative risk (RR) of an MI was calculated for both BB and EPI at each level of risk. Data was analyzed using SPSS.

RESULTS: Data was downloaded from the database July 15, 2004 and comprises 3745 consecutive acute pain patients. Duplicate patients were eliminated, 3662 operations were evaluated. There were 160 postoperative MI's of which $75 \%$ occurred with the first 48 hours of surgery. 568 patients received BB within 48 hours of surgery. 944 patients received a thoracic epidural. The RCRI was the only variable predicting POMI. Receiver operating characteristic for the RCRI was $0.75 \pm 0.02$. Both BB and EPI were more frequently used in higher risk categories. There was no reduction in the rate of POMI for beta blocker or epidural (RR at RCIC $>1$ was $0.85 \pm .35$ and $0.86 \pm .34$ respectively) Figure 1.

DISCUSSION: RCIC predicts POMI. The level of effect of BB in our analysis is consistent with other databases and much lower than proposed by Polderman or Mangano. At a 15\% reduction, for BB or EPI, this analysis is underpowered to show a significant effect. There is no effect in lower risk patients.

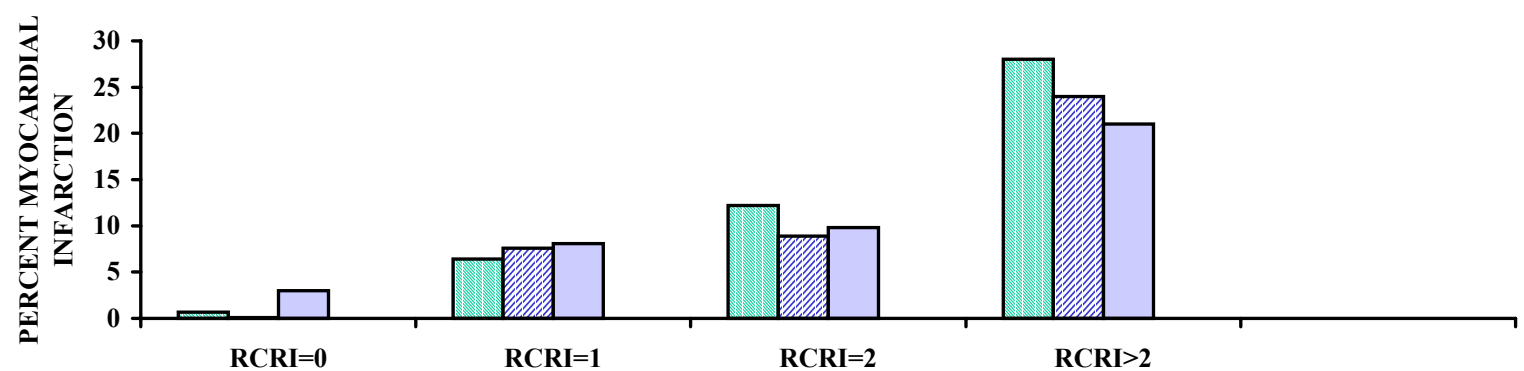

NO Rx שEPIDURAL $\square$ BETA-BLOCKER $\square$ 